\title{
Maria Venturi e Lorenza Ghinelli: Un ottimismo incauto?
}

\author{
Luciano Parisi \\ University of Exeter \\ I.parisi@exeter.ac.uk \\ ORCID identifier: 0000-0003-3132-6065
}

\section{Sommario}

Tra il 1987 e il 2017 Maria Venturi e Lorenza Ghinelli, autrici di grande successo commerciale, hanno scritto molte storie sull'abuso sessuale dei minori. In alcuni casi hanno mostrato una seria comprensione degli aspetti dannosi di una simile esperienza, ma in altri casi l'hanno descritta in modi che ne attenuano la traumaticità. Questo articolo riconosce l'aspetto incoraggiante che le storie di eventi dolorosi possono avere; segnala la difficoltà di trovare quell'aspetto nelle storie di abuso sessuale; sostiene che un incoraggiamento efficace non può essere trovato a spese di una rappresentazione realistica; e nota l'insolito valore che la verosomiglianza assume allora come criterio valutativo delle storie di abuso sessuale.

Parole chiave: Maria Venturi, Lorenza Ghinelli, letteratura italiana contemporanea, abuso sessuale sui minori, sofferenza, verosomiglianza

\begin{abstract}
Between 1987 and 2017, the best-selling authors Maria Venturi and Lorenza Ghinelli have written many stories regarding child sexual abuse. In some cases they have expressed a genuine understanding of the damaging aspects of such an experience, but in other cases they have underestimated and misrepresented its traumatic aspects. This article acknowledges the uplifting role that recounting traumatic events can have, notes that it is difficult to find this uplifting effect in the stories of sexual abuse and claims that an encouraging function cannot be achieved with unrealistic representations. It therefore stresses the unusual importance that verisimilitude has in our assessment of the stories of sexual abuse.
\end{abstract}

Keywords: Maria Venturi, Lorenza Ghinelli, contemporary Italian literature, sexual abuse of minors, suffering, verisimilitude 


\section{Maria Venturi e Lorenza Ghinelli. Un ottimismo incauto?}

The young women who contacted us needed accommodation and emotional support; they also desperately wanted images that reflected the reality of their own lives. ${ }^{1}$

Gli italiani discutono dell'abuso sessuale dei minori da molto più tempo di quanto, in genere, non ci si aspetti. I medici legali dell'Ottocento conoscevano quel che lo stupro significa per le vittime e non parlavano solo delle conseguenze fisiche della violenza carnale ma anche dello smarrimento, l'offesa e la vergogna. ${ }^{2}$ II codice penale Zanardelli, entrato in vigore nel 1890, stabiliva che i rapporti sessuali avuti da un adulto con minori di dodici, sedici o diciotto anni, a seconda dei casi, dovevano essere considerati atti di violenza anche quando gli abusanti non avevano esercitato forza fisica sui loro partner: quei giovani, infatti, non avevano ancora la capacità di prendere decisioni autonome in materia sessuale né la forza di dir no ad un adulto. ${ }^{3}$ L'Asilo Mariuccia, fondato a Milano nel 1902, tutelava le vittime di violenza sessuale e le ragazze costrette alla prostituzione (che erano spesso le stesse persone). Annarita Buttafuoco ha trovato negli archivi dell'Asilo carte che raccontano la vita di quelle ragazze e documentano lo stupore solidale e a tratti imbarazzato delle dirigenti a cui quelle vicende venivano narrate. ${ }^{4}$

Si sapeva dunque della diffusione dell'abuso sessuale, e si conosceva almeno una parte delle sue gravi conseguenze, ma la piena consapevolezza del fenomeno era in genere limitata ad ambiti ristretti. La violenza sessuale sui minori è stata compresa più ampiamente negli anni '70 del secolo scorso in Nord America e, nei decenni successivi, nella maggior parte dei paesi europei inclusa l'Italia. Le ragioni della svolta sono state molte: il benessere diffuso che permette di riservare maggiori attenzioni ai giovani, ${ }^{5}$ il lavoro svolto da pediatri come Henry e Ruth Kempe per la tutela dell'infanzia, ${ }^{6}$ la denuncia della violenza domestica fatta dal movimento femminista e la creazione di centri di assistenza contro lo stupro, ${ }^{7}$ la reazione di politici tradizionalisti preoccupati da quella che a loro pareva un'eccessiva libertà sessuale. ${ }^{8}$ La conoscenza pubblica è cresciuta soprattutto quando la stampa e i network internazionali hanno iniziato ad occuparsi di abuso sessuale dei minori in modo sistematico. Jenny Kitzinger denuncia la spettacolarizzazione dei reati che nel Regno Unito ha molte volte reso fuorviante l'azione di giornalisti e reti televisive, 
ma insiste anche sulla positività ultima del loro intervento: nonostante i limiti di molte pratiche informative, i media hanno aiutato il pubblico a capire il problema, favorendo la discussione di argomenti che prima erano considerati tabù. ${ }^{9}$

I testi letterari si inseriscono in questa storia e la riflettono. 'Libertà' di Giovanni Verga e 'Lucia' di Renato Fucini parlano di violenza sessuale sui minori alla fine dell'800. ${ }^{10}$ Autori di primo piano come Grazia Deledda, Luigi Pirandello, Federigo Tozzi, Paola Drigo, Alberto Moravia, Francesca Sanvitale e Dacia Maraini se ne sono occupati con insistenza nel corso del ventesimo secolo. ${ }^{11} \mathrm{Ma}$ l'argomento è stato più ampiamente trattato nei primi due decenni del ventunesimo secolo quando scrittori come Alessandro Baricco, Cristina Comencini, Maria Stella Conte, Elena Ferrante, Stefano Massaron, Laura Pugno, Gaia Rayneri, Antonio Scurati e Walter Siti hanno aumentato il numero dei temi trattati in relazione all'abuso ed introdotto variazioni importanti nei modi in cui se ne discute. ${ }^{12}$ Lo sfruttamento sessuale dei giovani è stato di volta in volta, e a seconda dei punti di vista, un'emergenza politica, un tema alla moda, un simbolo delle ingiustizie inevitabili in un'organizzazione patriarcale della società, un episodio tragico in molte vite che non si può né si vuole più tacere, un pretesto per paure collettive, ed altro ancora.

I testi dedicati all'abuso sessuale e alle sue conseguenze non formano a mio parere un genere o una categoria letteraria a se stante. Fanno semmai parte delle unfitting stories a cui Valerie Raoul ha dedicato nel 2007, come curatrice principale, un volume importante riferendosi a quei racconti di malati, disabili e vittime di traumi che parlano di come siamo chiamati a fronteggiare le limitazioni del corpo e della mente o una finitezza strutturale che prima o poi ci accomuna tutti. ${ }^{13}$ Che le storie di chi si trova in condizioni fisiche o psichiche problematiche (e si scopre unfit) siano state viste in molti periodi come sconvenienti (o unfitting) è una constatazione triste ma realistica. ${ }^{14}$ Nancy Mairs ha proposto un'altra definizione: 'literature of personal disaster' ${ }^{15}$ Anne Hunsacker Hawkins e Peter Kramer ne hanno suggerito un'altra ancora parlando di 'pathography' e 'autopathography'. ${ }^{16}$

All'interno di quelle storie, comunque si decida di chiamarle, i testi sull'abuso sessuale dei minori e sugli effetti che ne derivano costituiscono un filone tematico specifico che deve essere analizzato anche in quanto tale. Ha le sue componenti strutturali (l'abusato o l'abusata e i problemi che affronta, gli abusanti e le cause del loro comportamento aggressivo, la comunità che circonda l'uno e gli altri, e gli interventi che compie od evita di compiere, una serie di 
situazioni e temi ricorrenti) ed ha anche uno sviluppo storico pieno di svolte, con modelli narrativi di volta in volta emergenti che si scontrano nei periodi di transizione, con questioni diverse su cui si sono sviluppate riflessioni approfondite, e da cui in qualche caso sono derivate scelte od azioni significative.

\section{La necessità di un dialogo}

Questo articolo è dedicato alle storie di abuso sessuale sui giovani scritte da Maria Venturi (nata a Firenze nel 1933) e Lorenza Ghinelli (nata a Cesena nel 1981). Si tratta di due autrici nate a quasi cinquant'anni di distanza ma attive nello stesso periodo, ${ }^{17}$ ed accomunate soprattutto dal grande successo commerciale: Butta la luna $(B a)$, della prima, è presente in 573 biblioteche pubbliche e La colpa (Cp), della seconda, in $441 .{ }^{18}$ Fornendo questi dati, il catalogo generale delle biblioteche italiane segnala che, se qualcuno ha letto di recente una storia di abuso sessuale all'infanzia o l'adolescenza ambientata in Italia, ha letto probabilmente un testo dell'una o dell'altra scrittrice. ${ }^{19}$

Tutte e due hanno parlato di abuso in modi che sono stati a tratti strumentali. Si sono servite cioè di un tema improvvisamente privilegiato dai media per provocare con facilità l'interesse, il coinvolgimento e la commozione dei lettori. Attente per di più ai gusti del vasto pubblico, Venturi e Ghinelli sono state anche pronte ad assecondarne le tendenze deteriori semplificando spiegazioni, linguaggio e meccanismi psicologici per agevolare in quel modo la lettura dei propri testi. A volte assimilabili ai programmi della 'disturbingly uplifting prime time television' di cui parlano, in un altro contesto, Wulf Kansteiner e Todd Presner, ${ }^{20}$ quei testi sono stati relegati perciò nel ghetto dei 'popular subliterary genres' ${ }^{21}$

Le ragioni per prenderli comunque in considerazione sono tre. La prima è la necessità di discutere delle storie che circolano con successo nella sfera pubblica italiana. I valori essenziali di una società sono raramente espressi come concetti, ma sono per lo più trasmessi, discussi e modificati attraverso le storie che ci raccontiamo. ${ }^{22}$ Quelle che circolano più ampiamente contribuiscono alla soluzione dei problemi quando sono pertinenti ed intelligentemente impostate, e li aggravano negli altri casi. ${ }^{23}$ 'We have to choose carefully which stories to live with', osserva perciò Arthur W. Frank. ${ }^{24}$ Chi non conosce le storie raccontate da Venturi e Ghinelli - o 
le esigenze, conoscenze o pseudo-conoscenze che influenzano il pensiero e la condotta dei loro lettori abituali - si sottrae a un dialogo importante. Non partecipa, o partecipa solo frettolosamente, a un complesso processo collettivo di ridefinizione dei valori; e rinuncia alla possibilità di correggere equivoci diffusi.

La seconda ragione è che, misurandoci con queste storie e discutendone per accettarle, rifiutarle o per esprimere giudizi più seri e sfumati, siamo costretti ad esplicitare i nostri criteri di valutazione che non sono coerenti finché rimangono abbozzati. Nel contesto di questo filone tematico alcune domande richiedono risposte precise: che cosa cerchiamo in quel che leggiamo o ci raccontiamo? Esistono lettori o narratori le cui preferenze possono essere accantonate senza pregiudizio? A giustificare l'accantonamento basta il bisogno del lieto fine, così forte nei testi di Maria Venturi e in così forte contrasto con la negatività dell'abuso sessuale, specie quando è subìto nell'infanzia o nell'adolescenza? È lecito usare la verosimiglianza per valutare tali storie, visto che la validità di questo criterio è stata molte volte negata negli ultimi decenni in ambito letterario? Queste domande rimandano a temi dibattuti a lungo, in qualche caso per millenni, da un gran numero di persone sollecitate da ragioni diverse, all'interno di culture lontane l'una dall'altra, e non è possibile prendere una posizione che valga per ogni interlocutore, testo e cultura. Il mio obbiettivo è più modesto: vorrei mostrare come, discutendo delle opere di Venturi e Ghinelli, si raggiungono posizioni abbastanza chiare e convincenti per il filone letterario che ho preso in considerazione.

La terza ragione per analizzare i testi di Venturi e Ghinelli è che, facendolo, è più facile comprendere una sfida (e forse la sfida essenziale) che le storie di abuso sessuale pongono a chi le narra. Studiando i loro romanzi si fanno confronti (fra Venturi e Giacoma Limentani, per esempio, o fra Venturi e Alberto Moravia) che, quasi inevitabilmente, diventano contrapposizioni. ${ }^{25}$ Riflettendo su quelle contrapposizioni si scopre che dipendono per lo più dai modi in cui gli autori conciliano, da una parte, il realismo di fronte a sofferenze ai limiti della tollerabilità e, dall'altra, la ricerca di senso - oppure rinunciano a tale conciliazione. Venturi si limita per lo più all'affermazione di un senso a scapito di una rappresentazione onestamente realistica. Moravia è il suo opposto, limitandosi, soprattutto dagli anni '60 in poi, a una testimonianza tragicamente onesta ma negando ogni senso alle vicende che narra. Scrittrici come Elena Ferrante e Giacoma Limentani si collocano in posizioni intermedie che sono secondo me le 
più fruttuose. Le loro storie rientrano nella quest narrative di cui parla Frank con un ragionamento simile a quello che svilupperò (citandolo) nelle pagine che seguono. ${ }^{26}$

A queste tre ragioni si mescola anche il desiderio di contribuire alla definitiva rimozione di un pregiudizio che si manifesta ancora negli studi letterari italiani quando gli scrittori capaci di vendere centinaia di migliaia di copie di libri e i loro lettori sono confinati in una categoria inferiore; visti come creatori o fruitori di macchine gratificatorie che non assillano con questioni etiche, filosofiche o politiche; e contrapposti ai creatori e fruitori di romanzi seri, problematici e politicamente progressisti. ${ }^{27}$ Le diversità fra gli uni e gli altri sono più tenui e a volte impercettibili. La fede progressista, intanto, può a sua volta avere funzioni gratificanti fini a se stesse. ${ }^{28} \mathrm{Ci}$ sono poi problemi (sofferenze personali, malattie, la vecchiaia, la morte), che nessun progetto politico risolve in maniera definitiva e con cui è necessario confrontarsi anche in altri modi. ${ }^{29}$ Alcuni scrittori di successo hanno infine più doti di quanto si voglia in certi casi riconoscere come già Umberto Eco, sia pur con riluttanza, intuiva. ${ }^{30}$ Anche Venturi e Ghinelli, in certe parti delle loro opere, abbandonano l'approccio strumentale e le semplificazioni che l'accompagnano, mostrando un'approfondita consapevolezza degli effetti negativi dell'abuso. È un fatto di cui dobbiamo tener conto.

Se queste scrittrici sono ogni tanto in grado di affrontare temi difficili e ai limiti della trattabilità in un modo che può soddisfare critici esigenti, perché non lo fanno più spesso e sembrano invece riluttanti a presentare quelle pagine? Che cosa spiega le loro scelte? È probabile che siano i bisogni dei loro lettori o, quanto meno, dei lettori che si aspettano di avere. Anche quei bisogni vanno allora capiti e discussi, in ambito accademico ed extra-accademico, a meno che non si voglia equiparare sul serio il popolare e l'infraletterario, ed ignorare le aspettative di certi lettori perché la loro visione dell'esistenza, le loro illusioni o le loro speranze sulla redimibilità del mondo e il loro gusto letterario sono diversi, quando lo sono, da quelle dei critici letterari. In una cultura polarizzata come quella europea nella prima parte del ventunesimo secolo, che controlla a fatica le tendenze tribalizzanti accentuate dai social media, non c'è bisogno di ulteriori esclusioni ma, nel caso specifico, di un dialogo onesto fra diversi tipi di lettori. Ne vorrei perciò avviare uno sperando di avere argomentazioni capaci di coinvolgere lettori dal profilo diverso. Se queste argomentazioni saranno efficaci, e se ci sarà disponibilità all'ascolto, i lettori che Eco considera impegnati avranno la possibilità di riscoprire il sì alla vita che la 
narrazione di storie convoglia molto più spesso di quanto non abbiano generalmente notato. ${ }^{31}$ Altri lettori, quelli che Eco a torto considera disimpegnati, avranno invece la possibilità di rendersi conto dei vari tipi di sì alla vita che esistono, di quelli che reggono e di quelli che illudono solo, per poco tempo, e finiscono col fare più male che bene. Gli uni e gli altri matureranno così una maggiore tolleranza per le scelte culturali altrui, riservando i loro strali - se è necessario lanciarne - per i video-giochi violenti e le esibizioni rap che Laura Finley denuncia in Domestic Abuse and Sexual Assault In Popular Culture. ${ }^{32}$

\section{Maria Venturi e la fiducia nella vita}

Maria Venturi è nata a Firenze nel 1933, si è laureata in lettere, e ha lavorato a lungo come giornalista dirigendo le riviste Novella 2000 e Annabella. Per trent'anni ha curato una rubrica postale sulla rivista Oggi e due raccolte dei suoi saggi derivano dalle risposte alle lettrici di quella rivista. ${ }^{33}$ Venturi ha iniziato a scrivere libri di narrativa a metà degli anni '80, quando faceva anche la sceneggiatrice per la RAI e molti suoi romanzi sono diventati film o serie televisive. ${ }^{34}$ La trama e lo stile di quei romanzi sono influenzati dalle altre attività della scrittrice: la corrispondenza con le lettrici suggerisce figure, situazioni e temi; la possibilità di trasformare i testi in produzioni televisive impone 'una trama "forte" e densa di accadimenti'; 35 la pratica giornalistica incoraggia una lodevole varietà di ambienti, un sostenuto sforzo documentario, ${ }^{36}$ la descrizione sintetica ma precisa dei caratteri, e una parziale semplificazione delle questioni. Si aggiunga a tutto questo l'esigenza del lieto fine: la scrittrice conosce il suo pubblico, prevalentemente femminile; sa che le sue lettrici si immedesimano coi personaggi, ${ }^{37}$ che hanno come loro ostacoli da superare, desideri da realizzare, ambizioni da soddisfare; e vuole mandare il messaggio incoraggiante che ogni obbiettivo, o quasi, può essere raggiunto. Il lieto fine 'è di rigore', dice: 'una forma di ringraziamento per chi ha scelto di leggere il suo libro'. ${ }^{38}$

La scrittrice incoraggia alla fiducia nella vita in modi che a volte sono sensati e persino ammirevoli ('metà delle sofferenze se eviterebbero sensa lo orgoglio', dice con italiano stentato un suo personaggio'), ${ }^{39}$ ma che in altri casi portano a sottovalutare pericolosamente la dimensione dei problemi. Questa sottovalutazione è evidente nelle storie di Joanna Fusaro ne $L a$ 
moglie nella cornice $(M c),{ }^{40}$ e di Sabrina Nardi in Butta la luna. Joanna perde i genitori a nove anni, subito dopo essere stata violentata da un cliente della madre. In orfanotrofio rifiuta il cibo. A quindici anni è anoressica e pesa trenta chili. Venturi sa che le conseguenze di un'infanzia devastata durano tutta la vita, ${ }^{41}$ che lasciano delle ferite gravi, ${ }^{42}$ e dice esplicitamente che una giovinezza come quella di Joanna 'avrebbe disintegrato qualunque essere umano'. ${ }^{43}$ Ma Joanna non si disintegra: si riprende anzi in poche pagine e, a ventitré anni, è 'la top model del giorno' ${ }^{44}$ Ha sentimenti generosi, buon gusto, buon senso, successo, autonomia finanziaria e psicologica, e nel corso del romanzo mostra di essere una donna 'realmente forte'. ${ }^{45}$ È tutto troppo facile, in maniera persino offensiva per chi conosce questi problemi e sa quanto sia complicato anche solo mitigare la disapprovazione di se stessi, il senso di fragilità e d'impotenza prodotti dall'abuso in giovane età. ${ }^{46}$

Sabrina viene avviata alla prostituzione dalla madre quando ha cinque anni. Separata dalla famiglia ed istituzionalizzata, rigetta i valori delle educatrici e, compiuti i diciott'anni, torna dalla madre per far di nuovo la prostituta. Due persone cambiano la sua vita: un'assistente sociale fa emergere in lei un desiderio di cambiamento, e un'imprenditrice (madre dell'assistente sociale) le offre un lavoro. La giovane diventa attiva, equilibrata e generosa. Quel cambiamento non matura lentamente nella coscienza o nella psiche, e non è neppure raccontato come fiaba, mito o visione. La metamorfosi di Sabrina è presentata come un evento realistico, e il riferimento alla realtà suscita le obiezioni più serie: chi è stato vittima di violenza sessuale da bambino o da adolescente ha patito a causa di alcuni dei peggiori comportamenti umani; perde la capacità di 'fidarsi e affidarsi'; 47 ed è difficile che riesca ad instaurare sani rapporti interpersonali. ${ }^{48}$ È 'come $\mathrm{se}^{\prime}$, dice Malacrea, 'in un mondo reso nemico dal tradimento primario ricevuto dall'abusante, tutto piombasse in un silenzio artificiale e deformante' ${ }^{49}$ Le affermazioni di principio di Venturi sono come sempre compatibili con (e derivate da) quelle di psichiatri e operatori sociali: 'I'amore non bast[a] per fa[r] superare il trauma né per reinserir[e] in un contesto normale' (Ss, p. 177); 'I'amore non basta per salvare l'amico o il fratello o il figlio' (Ip, p. 243). E allora? come può una giovane sfruttata da quasi ogni adulto che ha conosciuto fidarsi all'improvviso del prossimo? Quali sono le conquiste che l'aiutano? Quali le reazioni alle inevitabili delusioni? 
La scrittrice non pone queste domande perché il sapere specialistico di chi aiuta le vittime di abuso, che lei ha studiato e capito, si scontra con l'istinto della narratrice e con quello che sa (o immagina) dei suoi lettori più fedeli. Un personaggio de La storia spezzata (Ss) afferma: 'con buona pace del tuo Freud, penso che l'ottanta per cento della psicologia sia solo elementare buonsenso. E non occorre una laurea, per possederlo'. ${ }^{50}$ L'elementare buon senso' della scrittrice la spinge a cercare un lieto fine anche in un posto così poco adatto come i romanzi sull'abuso sessuale. Quell'abuso, però, come dice Malacrea, è paragonabile 'a una bomba a orologeria, di cui nessuno conosce e controlla il timer, pronta a dilaniare nel presente e nel futuro con sofferenze e fallimenti pesantissimi' ${ }^{51}$

L'errore di fondo di Venturi non è la ricerca di qualcosa di consolante. Coloro che parlano di malattie o traumi che sconvolgono la fiducia di un essere umano nel mondo, negli altri o nel proprio corpo sentono il bisogno di confrontarsi con quei mali. ${ }^{52}$ Registrano la crisi e vogliono sottrarsi, o sottrarre altri, alla marginalizzazione che comporta. Cercano parole, gesti od azioni da opporre alla vulnerabilità che paralizza ed isola. Si augurano che chi è responsabile del trauma (se qualcuno lo è) sia assicurato alla giustizia. Possono affermare l'insensatezza di tanta sofferenza, ma una simile conclusione è il risultato di una ricerca inizialmente animata da ben altri obbiettivi. In questo senso l'impostazione di Venturi è autorevolmente condivisa. I grandi testi letterari dell'Ottocento europeo sull'abuso sessuale dei minori - Delitto e castigo (1866) e I demoni (1871) di Fëdor Dostoevskij, 'La petite Roque' (1885) di Guy de Maupassant - si concludono con il suicidio dei personaggi abusanti, come se gli autori volessero garantire ai lettori che giustizia è stata o sarà in qualche modo fatta. Non sono eventi lieti, i suicidi, ma riconciliano in questi casi la storia di una violenza esecrabile con quello che si crede, si vuole, e conta nella nostra cultura. Introducono un elemento di positività.

Anche le scrittrici italiane che nel ventesimo e ventunesimo secolo hanno narrato con intelligenza storie di adolescenti abusate hanno lottato di continuo per conciliare, da una parte, l'esigenza di una documentazione onesta e dunque desolante - la Maria Zef di Paola Drigo ha fin dall'inizio, nei confronti dello zio abusante, un atteggiamento 'di soggezione e di umiltà', la protagonista della Trilogia di Giacoma Limentani rivive per decenni l'orrore della violenza subita - e, dall'altra, il bisogno di illuminare i casi delle loro protagoniste con un momento redentivo.$^{53}$ 
Dopo grandi sofferenze, Maria Zef si ribella a chi abusa di lei. Lo fa con azioni su cui storiografi, giuristi e psichiatri esprimono perplessità ma di cui non negano alla fine la possibilità. ${ }^{54}$ Mina, la protagonista della Trilogia, racconta la violenza subita, denuncia i colpevoli, ottiene la giustizia che cercava, supera gli effetti sconvolgenti del trauma patito, ma lo fa a fatica, in tarda età, in modo sereno e disincantato insieme. La Delia de L'amore molesto di Elena Ferrante si trova nella stessa posizione di Mina quarant'anni dopo aver patito gravi molestie sessuali e provato sensi di colpa laceranti e, a conti fatti, immeritati. Venturi è diversa da quegli scrittori e queste scrittrici perché di rado sfuma le proprie storie. Il suo errore di fondo è quello di presentare tenui spiragli di speranza come se fossero certezze o verità scontate; di trasformare in lieto fine il gesto, il pensiero, l'attimo consolante. La convinzione infondata che il narratore abbia una particolare competenza nella materia trattata, quando c'è, fa sì che quell'errore abbia influenze particolarmente negative su alcuni lettori. ${ }^{55}$ In ultima analisi, l'errore di Venturi è uno di quelli che spingevano Platone a condannare la (presunta) mimesi artistica per la disinformazione che può produrre e per la contaminazione della realtà che in quei casi comporta. ${ }^{56}$

Secondo lo psicologo Jerome Bruner, una buona storia ha sempre le due caratteristiche a cui mi sono riferito: è credibile, e dà senso a quel che narra ('[it] endow[s] experience with meaning'). ${ }^{57}$ Essere credibili, per Bruner, significa raccontare una storia che corrisponde alle aspettative di base di chi appartiene alla cultura dell'autore o dell'autrice, o che permette di capire perché qualcosa avviene in un modo che non ci si potrebbe altrimenti spiegare. Dare un senso, secondo Bruner, equivale a confermare presupposti diffusi ma impegnativi e spesso accettati inconsciamente: che il mondo ha una sua leggibilità e narrabilità, che possiamo trovare una collocazione nella sua struttura, che siamo in grado di fare scelte e che possiamo agire per provare ad ottenere quello a cui miriamo. Le stesse caratteristiche sono segnalate come essenziali in ambito narratologico, storico, e filosofico. ${ }^{58}$ La romanziera Rachel Cusk si riferisce a loro quando sostiene che una buona storia è vera e bella. ${ }^{59}$ II critico letterario Thomas Couser preferisce parlare, da una parte, delle 'demands of truth and vigilance' e, dall'altra, del 'need for closure' (chiusura della crisi, superamento degli ostacoli all'autoaffermazione dei protagonisti). ${ }^{60}$

Le storie di abuso sessuale commesse sui minori contrappongono molte volte una delle due caratteristiche all'altra: sembrano prive di senso, o sembra quanto meno che parlino di un 
mondo senza senso, quando sono credibili; e sembrano per lo più incredibili quando suggeriscono un senso. Le storie di Moravia appartengono con rare eccezioni al primo tipo. I loro particolari anticipano con precisione sorprendente le analisi dei più brillanti psichiatri contemporanei. Su quella base, però, Moravia arriva alla conclusione, sofferta ma perentoria, che non c'è nessun rimedio al trauma dell'abuso: Andreina e Carlino ne Le ambizioni sbagliate (1935) e tanti altri suoi personaggi sono destinati a restare infelici ed inquieti per sempre. ${ }^{61}$ Niente redime la loro vita. Come dice un personaggio di lan McEwan, 'who would want to believe that, except in the service of the bleakest realism?'62 Nel filone letterario che mi sta a cuore i romanzi di Venturi rappresentano l'estremo opposto. Sono pieni di ottimismo. Suggeriscono che l'abuso sessuale è una prova dura, che può essere peraltro affrontata con successo. Superando in tempi brevi ostacoli sgomentanti, le eroine di quei romanzi suscitano però la netta incredulità di chi sa qualcosa di abuso sessuale, e i dubbi degli altri lettori che non sono sciocchi e intuiscono la natura sconvolgente di ogni abuso. ${ }^{63}$ Le storie di Venturi, così, non soddisfano il primo dei requisiti menzionati da Bruner. Moravia e Venturi rappresentano estremi contrapposti e di diversa qualità. Ma la sfida - superata o no - che accomuna chi scrive queste storie è quella di essere allo stesso tempo convincenti (e non sminuire le pesantissime conseguenze dell'abuso sessuale) e capaci di trovare un senso nelle vicende narrate o di dargliene uno (per quanto remoto o sfuggente possa essere). È quello che il filosofo Theodor Adorno chiama il paradosso di un'arte a cui non è lecito ingannare e da cui, in genere, ci si aspetta anche una parola pacificante quando è alle prese con sofferenze smisurate, che proibiscono e richiedono allo stesso tempo la sua presenza. ${ }^{64}$ Superano la sfida, a mio parere, scrittrici come Drigo, Limentani e Ferrante che si muovono fra un estremo e l'altro senza mai rinchiudersi in uno, che parlano della severità dei traumi ma anche di un loro non sempre possibile, quasi sempre parziale, e tuttavia (in qualche modo, per alcuni) convincente superamento. La nostra vita, dice Frank, che su questi temi offre sempre indicazioni decisive, è un tentativo di dar senso a quel che ci tocca testimoniare, ma siamo molto limitati dalla datità. ${ }^{65}$

Venturi ammette il proprio fallimento ne La bambina perduta $(B p)$. In quel romanzo insiste più che altrove sugli effetti negativi che la violenza sessuale ha sulle bambine. Paola era 'combattiva e fantasiosa' ma dopo uno stupro diventa 'incapace di comportamenti reattivi' e la 
sua fantasia si atrofizza; ${ }^{66}$ i rapporti con gli altri si complicano; ${ }^{67}$ i sensi di colpa la perseguitano. Eppure, nonostante tanti avvertimenti, nella trama che Venturi racconta, la protagonista non risente di queste limitazioni. La mancanza di fantasia non le impedisce di trovare 'un'autoterapia istintiva'. ${ }^{68}$ La difficoltà di rapportarsi con gli altri non crea ostacoli al suo innamoramento. ${ }^{69}$ L'incapacità di comportamenti reattivi scompare dopo l'incontro con l'uomo della sua vita. ${ }^{70}$ Le contraddizioni fra quello che il romanzo afferma e quel che mostra sono così evidenti che la scrittrice, per rispetto dei propri lettori e, credo, anche per rispetto delle vittime di abuso, lo interrompe dopo 140 pagine scarne, che ne fanno di gran lunga il più breve della sua produzione. $^{71}$

Ci sono tuttavia occasioni in cui Venturi accetta la lezione che il suo lavoro documentario le ha permesso di raccogliere e propone le sue storie senza correzioni ottimistiche, con un atteggiamento fondamentalmente interrogativo. In quelle occasioni scrive testi degni di rispetto: penso in particolare alle storie di Fiorenza in Incantesimo (In), e di Fabrizio in Butta la luna. Violentata a quindici anni, Fiorenza si procura un lavoro e va a vivere con il figlio in un istituto religioso. Quando il suo abusatore, incarcerato per un diverso delitto, viene rilasciato per buona condotta e un avvocato senza scrupoli gli permette di recitare la parte del 'povero innocente perseguitato', la giovane si uccide buttandosi dal terzo piano dell'istituto, dove pure era al sicuro da lui. ${ }^{72} \mathrm{Nel}$ contesto ottimistico di questi romanzi, il gesto è inspiegabile. La scrittrice attenua lo shock dicendo che la vita di Fiorenza 'era troppo dura, senza alcuna speranza', ma non prova neppure a risolvere il mistero: 'qualcosa è scattato nella sua mente. Repulsione? Terrore? La sola cosa certa è che ha deciso di uccidersi' ${ }^{73}$ Scatta il timer di cui parla Malacrea; l'equivalente psicologico di una bomba esplode nella mente di una persona, la dilania e la devasta.

Fabrizio, in un romanzo scritto quattro anni dopo Incantesimo, vive in un istituto di accoglienza per adolescenti sottratti alla potestà dei genitori. È chiuso al dialogo, abituato a simulare, quieto in apparenza ma animato da una rabbia profonda per i torti subiti. Dato in affidamento, uccide una bambina di tre anni adottata dalla stessa famiglia simulando un'incursione di estranei. Quando l'avvocato lo informa che una prova è stata trovata contro di lui, si chiude in camera e si uccide. L'uomo che lo ha in affidamento scopre il cadavere al mattino: 
solo in quel momento ebbe la certezza che era colpevole. Pensò alla moglie che dormiva ignara nella stanza accanto, alla figlia Emma che prima di morire aveva conosciuto tutta la paura e il dolore del mondo: Fabrizio aveva devastato le loro vite. Che cosa hai fatto? L'urlo gli esplose nelle viscere e fu sommerso dall'incredulità e dall'odio. ${ }^{74}$

La storia è cupissima. Ricorda per questo L'attenzione (1965) e La vita interiore (1978), due romanzi di Moravia dedicati a casi desolanti di minori sessualmente abusati. ${ }^{75}$ Per una volta, però, I'infraletteraria Venturi supera un maestro della letteratura internazionale esprimendo uno sbigottimento genuino e reagendovi con una forza di cui quei due romanzi moraviani sono privi. L'interlocutore di Baba, ne L'attenzione, e quello di Desideria, ne La vita interiore, non mostrano pietà per quel che accade alle protagoniste di quei romanzi, ed esibiscono semmai un'inquietante curiosità. Anche Cosima, l'assistente sociale che ha seguito Fabrizio, non prova pietà per lui, almeno per i primi giorni, ma s'interroga angosciata sul proprio rapporto con il ragazzo:

ti senti in colpa per non averlo saputo aiutare? È perché, rifiutando il tuo aiuto, ha attentato alla tua autostima e ferito la tua sublime vanità professionale? È perché non riesci a provare altra pietà se non [...] per la povera bambina massacrata? Cosima si soffermò, meticolosamente e lucidamente, su ogni interrogativo e soltanto alla fine vide chiaro in se stessa. La pena per Fabrizio era raggelata dall'incapacità di capire che cosa lo aveva spinto a uccidere [...]. Non riusciva $a$ darsi le risposte. ${ }^{76}$

Alla fine Cosima si rende conto di un particolare importante: Fabrizio si è confidato con qualcuno. La prova che ha permesso di incolparlo è stata trovata grazie a una lettera anonima spedita da chi conosceva i dettagli dell'accaduto. A scrivere quella lettera, scopre l'assistente sociale, è stata I'unica persona a cui Fabrizio voleva bene, la sorella:

è stata Sabrina a scrivere la lettera anonima. In quella denuncia, apparentemente crudele, Cosima scorgeva l'unico sprazzo di luce nel buio di quella tragedia. Fabrizio, incapace di sopportare da solo il peso di quanto aveva fatto, aveva cercato di condividerlo con Sabrina. E Sabrina, incapace di tenere per sé quel terribile segreto, aveva scritto ai carabinieri. Era il segno confortante che qualcosa di buono, nei due fratelli, era sopravvissuto alle violenze, al deserto di valori, al marciapiede. [...] infine arrivò anche la pietà per Fabrizio. ${ }^{77}$ 
Venturi supera qui la sfida essenziale che le storie di abuso sessuale dei minori pongono a chi le scrive. Riesce anche lei a conciliare l'esigenza di una documentazione onesta ma sconsolante, e il bisogno di illuminare le vicende: sentendo rimorso per la propria azione, l'incomprensibile, furioso Fabrizio ha accettato almeno una regola base della nostra comune umanità (o di quello che riteniamo tale).

Molte domande in Butta la luna restano senza risposta ma in questo caso, a differenza di quel che accade nella storia di Sabrina raccontata nello stesso libro, sono poste, ${ }^{78}$ e Venturi non risponde per umiltà conoscitiva. mostrando la stessa malinconia con cui racconta la storia di Carlotta D'Angelo in Punta di cuore, ${ }^{79}$ o l'autodistruzione di Laura ne L'amore stretto, ${ }^{80}$ e parla del sadismo di chi 'ferisce, rimprovera, colpevolizza, ignora e maltratta per toccare con mano fino a che punto può spingersi'. ${ }^{81}$ Su questi aspetti sconsolanti della vita, però, Venturi si sofferma malvolentieri. La trama di Butta la luna è rivelatrice: dopo aver trattato stupita degli eventi tragici che compongono l'esistenza di Fabrizio, la scrittrice si sbilancia in direzione opposta per ravvivare lo spirito dei lettori con la storia eccessivamente rassicurante della conversione di sua sorella Sabrina. È una fuga in un ottimismo incauto, nell'illusorio: il cedimento, direi, a una tentazione pericolosa.

A giustificazione di Venturi e delle proprie inclinazioni, i suoi lettori potrebbero osservare che è facile avere simpatia per chi cede a quella tentazione, molto più che per chi non l'avverte neppure. Potrebbero aggiungere che auspicare in modo maldestro il bene o la giustizia è meglio che darne l'assenza per scontata. ${ }^{82}$ Temo però che non sia così: alcune vittime di abuso si illudono di essere amate, sia pur inadeguatamente, da chi invece si serve di loro e non riescono anche per questo a staccarsene; ${ }^{83}$ altre si illudono che le sofferenze possano scomparire purché si pensi ad altro. Le illusioni propagano l'infelicità a cui si vorrebbe rimediare ricorrendo ad esse: possono servire come distrazioni, e bisogna comunque rispettare chi ci si affida, ma accettarle è probabilmente un segno di resa.

\section{Lorenza Ghinelli e la tentazione della violenza.}

La produzione letteraria di Lorenza Ghinelli presenta oscillazioni simili a quelle che appaiono nei romanzi di Venturi. Nata a Cesena nel 1981, la scrittrice ha una laurea in scienze della formazione, 
un master in tecniche della narrazione, e ha a sua volta lavorato come sceneggiatrice per Taodue e Canale Cinque. ${ }^{84}$ I libri di Stephen King hanno avuto qualche influenza sulla sua opera, ${ }^{85}$ ma le tecniche narrative di Ghinelli sono collegate soprattutto a musica e televisione. Le citazioni più efficaci nei suoi testi riguardano brani musicali: degli Afterhours ('Sui giovani d'oggi ci scatarro su', 'Voglio una pelle splendida', 'Dentro Marilyn'), di David Bowie ('Cygnet Committee', 'Heroes'), i Clash ('Tommy Gun', 'Give 'em Enough Rope', 'Safe European Home') e dei Sex Pistols ('Who Killed Bambi?', 'I Wanna Be Me'). ${ }^{86}$ La struttura delle sue storie riproduce quella dei serial televisivi: ogni puntata o segmento narrativo inizia con una scena d'azione e un problema da risolvere; e, quando il problema trova la sua soluzione, la puntata o il segmento narrativo finisce annunciando un problema nuovo. ${ }^{87}$ Ghinelli scrive storie dense di eventi e di scelte che $i$ personaggi fanno, reagendo a quel che accade. Nei romanzi di Venturi l'elemento rassicurante è l'esito della storia. Nei romanzi di Ghinelli lo è invece la volontà di autoaffermazione, la presenza di quella che in inglese viene chiamata agency. La capacità reattiva dei personaggi di Ghinelli viene presentata come una forma di agency, anche quando le scelte fatte sono sbagliate. Un tale approccio permette alla scrittrice di narrare con maggiore facilità storie di sconfitte, perché queste possono essere riconosciute come tali senza compromettere un generico ottimismo di fondo.

Nel primo romanzo di Ghinelli, J.A.S.T., del 2010, speculazioni finanziarie e politica internazionale creano lo sfondo a una fitta serie di avventure. Fra i personaggi ci sono Gheddafi e Osama bin Laden, e fra i temi trattati l'abuso dei minori nel Terzo Mondo. Un agente dei servizi segreti israeliani in servizio a Kabul è tentato da una prostituta dodicenne; e la piccola, candida, affettuosa Aisha viene data in sposa a un rozzo e crudele mercante. ${ }^{88}$ Aisha ne subisce le violenze sessuali per quattro giorni. Poi, cambiando bruscamente carattere, lo sgozza con un rasoio, ${ }^{89} \mathrm{e}$ fugge da Kabul facendo strage di talebani. L'energia fisica che la sorregge è improbabile, e segnala una mancanza di riflessione in Ghinelli che sembra credere - come il crudele marito di Aisha, come gli aggressivi talebani del romanzo - che il mondo appartiene ai più forti: i giusti trionferanno se saranno in grado di procurarsi coltelli più affilati di quelli dei loro avversari, e se sapranno usarli con maggiore spregiudicatezza, anche a costo di non essere più giusti. La scrittrice è impegnata in un esordio precoce, e la sua scarsa esperienza spiega in parte quest'errore di prospettiva. 
La scrittura di Ghinelli è a volte irritante, ma ha una sua logica. Delinea un ambiente, un carattere, o riassume il senso delle situazioni con poche parole e molte ripetizioni: il Martino de La colpa, per esempio, viene violentato dallo zio e 'questo momento, questo adesso, questo ora, ficca tutti gli ieri del mondo dentro a una cassa. La chiude, la inchioda, la interra. E senza un amen si sfregia tutti i domani del mondo'. ${ }^{90}$ II lessico è spesso volgare, ma può essere creativo: in una notte di rimorsi la luna è 'glauca, lattiginosa, malatissima', ${ }^{91}$ e nei giorni di festa gli anziani clienti del bar Nizza retrocedono 'sotto il dehor, al riparo della perturbante gioventù' ${ }^{92}$

I protagonisti dei romanzi posteriori sono adolescenti o tardo-adolescenti dell'Italia di oggi. Sono vulnerabili, minacciati, perseguitati da coetanei ed adulti. Le loro storie includono presto, e con una certa naturalezza, casi di abuso sessuale che hanno un ruolo ampio ne La colpa e in Con i tuoi occhi (Ct)..$^{93}$ I personaggi di spicco ne La colpa (del 2012) sono tormentati tutti da un senso di colpa. Ghinelli descrive con perspicacia la loro pensosità, irritabilità, malinconia e riluttanza all'affetto. ${ }^{94}$ Non dice molto, però, sulle cause di quei sensi di colpa e su come sono vissuti: 95 Martino è perseguitato dal ricordo di un abuso rifiutato con eccessiva debolezza e poi subito passivamente; Estefan sospetta di aver fatto del male a un fratello e di aver rimosso quell'azione dalla memoria ;ma le loro personalità, i loro comportamenti, le loro forme di aggressività sono talmente simili che un editor tardivo potrebbe scambiare i loro passati senza sbilanciare l'equilibrio del romanzo. Si sarebbe tentati di dire che le conoscenze della scrittrice sono ancora una volta limitate, anche a causa di una disinformazione generalizzata che sul problema si ha in Italia. ${ }^{96}$

Eppure, in Con i tuoi occhi (del 2013), la scrittrice parla con improvvisa competenza. Le ragazze abusate in questo romanzo sono Maya e Irma. La prima è una ragazza di provenienza afghana, priva di interessi, 'perennemente intenta a masticare gomme', ${ }^{97}$ che di notte, quando i suoi genitori dormono, si allontana da casa per soddisfare i desideri sessuali di tre adulti che hanno 'plasmato e piallato [il suo carattere] adeguandola al proprio immaginario'. ${ }^{98}$ Irma assiste a quelle orge e ne è sconvolta. Maya sembra contenta. Freme dalla voglia di mostrare all'amica quanto è brava e implora che la 'scop[ino] di più e di più ancora': 99

[era] trasfigurata dallo sforzo e dal compiacimento. La caricatura di se stessa. Le sorrise complice, le disse qualcosa con gli occhi, qualcosa che Irma non seppe tradurre. Poteva essere un: hai visto quanto sono brava? Oppure un salvami. Forse 
un guardami e dimmi che sono quello che vedono loro. Dimmi che mi invidi. Dimmi che vuoi essere come me. $O$ forse era solo uno sguardo di vetro, e dietro quegli occhi non c'era niente. ${ }^{100}$

La ragazza afghana si ammala di sifilide, viene chiusa in riformatorio, scappa, resta incinta e scompare dal romanzo lasciando dietro di sé l'enigma di quello sguardo.

Trascurata dai genitori, Irma segue gli insegnamenti superficiali di alcune coetanee. Pensa che i maschi siano solo interessati al sesso, che i sentimenti non contino. A dodici anni si veste in modo provocante; a quindici ha un'intensa vita sessuale; a venti considera malinconica 'i corpi che ha conosciuto' e che, 'in fondo, sono tutti uguali'; ${ }^{101}$ a ventinove dipende da alcol e droghe, incapace di tirare avanti. Tornando a casa di sera, un uomo la vede per terra malconcia: prova pietà, la porta a casa, le fa passare la notte nella camera di una figlia in vacanza.

Quella camera colpisce Irma perché racconta una vita 'anni luce distante da quella che [lei ha] condotto credendola l'unica possibile'. ${ }^{102}$ Vorrebbe tornare 'indisturbata alla vita di sempre' e invece risolve proprio allora l'enigma dello sguardo di Maya. Capisce di avere, nei confronti della figlia del suo soccorritore, l'atteggiamento che Maya aveva avuto anni prima per lei: l'invidia, e dietro l'invidia c'è la consapevolezza di vivere una vita sbagliata, senza le soddisfazioni che sarebbe legittimo aspettarsi.

Le situazioni più importanti nel movimentatissimo finale del romanzo sono due. La prima suggerisce la possibilità di un riscatto in Irma che

avverte qualcosa di nuovo, anzi, di vecchissimo agitarsi nel suo petto. Qualcosa che evoca universi di un tempo perduto antecedente a Maya, alla mercificazione dei corpi e all'oblio. Qualcosa di ammaccato, sconvolto, tumefatto, ma nonostante tutto ancora inviolato. L'idea che possa essere rimasto qualcosa da salvare la riempie di terrore. Ma è un sentimento preferibile al deserto, al nulla che riempie di niente. ${ }^{103}$

La seconda suggerisce che l'amore, sempre frainteso e sostituito con 'il placebo dei corpi', ${ }^{104}$ possa nascere dalla relazione che Irma ha con un'altra donna. La giovane sa solo di essere 'un casino', di aver paura, di voler cambiar vita e le sue speranze sono credibili proprio per la loro esilità, ${ }^{105}$ allo stesso modo in cui le carezze incerte che scambia con l'amante sono più forti e convincenti del rasoio con cui I'Aisha di J.A.S.T.sgozzerebbe i combattenti islamici per le strade 
delle città afghane. Un'autrice come Ghinelli, che predilige i ritmi veloci, l'azione serrata, i colpi di scena che coinvolgono città, nazioni, e il nostro intero pianeta, scopre all'improvviso i momenti apparentemente insignificanti di intesa fra sconosciuti, $i$ 'rapporti misteriosi che s'esprimono in un sorriso inavvertito e nello sguardo', ${ }^{106}$ che non si possono spiegare e che fanno però rivivere anche fra gli adulti un'allegria spensierata e quasi infantile, dando energie inaspettate. In alcune pagine conclusive di Con i tuoi occhi, anche Ghinelli concilia l'esigenza di una documentazione desolante con il bisogno di illuminare con scintille isolate una storia d'abusi. Le vendette e i trionfi all'improvviso non hanno più rilevanza. Per dare senso a una storia o una vita basta - ed occorre - molto meno. ${ }^{107}$

\section{Conclusioni}

Nelle opere di Maria Venturi e Lorenza Ghinelli si alternano così una tendenza alla semplificazione, alla formula e all'ottimismo di maniera, da una parte, e la capacità di superare gli stereotipi per raccontare l'abuso sessuale dei minorinella sua desolante realtà, dall'altra. Lo sviluppo cronologico della loro opera e i pochi dati biografici disponibili non spiegano le oscillazioni che ho messo in evidenza e la preferenza accordata al modello ottimista e formulaico. Mi pare significativo però quel che accade in Butta la luna e in Con i tuoi occhi. Subito dopo aver raccontato la storia di abuso sessuale più convincente della sua intera carriera letteraria, quella di Fabrizio Nardi, Venturi la integra con quella altamente improbabile di sua sorella Sabrina. Subito dopo aver rappresentato i minimi, appena percepibili, progressi di Irma, Ghinelli conforta i lettori con una serie mirabolante di colpi di scena. Una rappresentazione genuinamente realistica è in molti casi un requisito troppo pesante per raggiungere l'obbiettivo rassicurante che Venturi, Ghinelli, e i loro lettori in genere si propongono. Come nota Bruno Bettelheim, incoraggiamenti realistici, cauti, e moderati possono avere effetti più deludenti che consolanti. ${ }^{108}$

Analizzando questi libri, si comprende bene - e si può far vedere bene ad altri - qual è una sfida essenziale che incombe su chi narra queste storie (essere credibili, evitando forme di pessimismo assoluto) e quali sono i modi, i momenti, e le condizioni in cui la sfida è affrontata, quali le semplificazioni che è bene evitare, quali le incertezze che è saggio ammettere. Anche altre vie portano a quella comprensione, ma questa è rapida, chiara, e percorsa abbastanza 
spesso: studiando le narrazioni dell'olocausto, anche Kansteiner e Presner sentono il bisogno di misurarsi con opere 'disturbingly uplifting' da cui prendono poi, cautamente, le distanze.

Analizzando i testi di Venturi e Ghinelli si chiariscono il ruolo dei lettori e vari criteri di valutazione utili nel filone letterario che ci interessa. Romanzi come La bambina perduta, della prima, e La colpa, della seconda, richiedono interpreti sospettosi, lettori che interrogano il testo, ne rivelano le contraddizioni, ne smascherano le immagini convenzionali, ne destabilizzano I'illusoria visione di fondo. Le storie di Fabrizio e di Irma meritano invece interpreti cooperanti: ${ }^{109}$ presentano sforzi di comprensione che chi legge può imitare, integrare con profitto e magari sentire il bisogno di sostituire con sforzi meglio impostati e atteggiamenti di più profonda solidarietà, ma che sono comunque fattivi. Le stesse scrittrici raccontano La bambina perduta, La colpa e le storie di Fabrizio e di Irma: è dunque bene che ogni lettore abbia la capacità di adottare al momento opportuno atteggiamenti di dissenso e di cooperazione senza mai presupporre la qualità, o l'assenza di qualità, in quel che legge. Una posizione genuinamente critica è vigile, flessibile, incompatibile con preclusioni pregiudiziali e generalizzazioni.

I criteri che ho usato qui per valutare o svalutare i diversi romanzi sono stati principalmente due, intrecciati fra di loro: quello della logica interna ('come può una giovane sfruttata da quasi ogni adulto che ha conosciuto fidarsi all'improvviso del prossimo?'; 'la difficoltà di rapportarsi con gli altri non crea ostacoli al suo innamoramento?') e quello del riscontro col reale ('il riferimento alla realtà suscita le obiezioni più serie'). Non mi pare che il primo criterio sia controverso. ${ }^{110}$ II secondo è stato guardato invece con sospetto perché, dice Tzvetan Todorov, I'importance [des mots] dépasse celle des choses qu'ils étaient censés refléter', ${ }^{111}$ e, come dice Pernot, il 'verosimile' è diventato in troppe occasioni 'le dogmatisme d'une parole critique qui impose [...] une idéologie'. ${ }^{112}$ II concetto stesso di 'vero' suscita perplessità in ambito letterario. ${ }^{113}$ Ma bisogna stare attenti a non confondere verità di ragione e verità di fatto, ragionamenti deduttivi e osservazioni empiriche. II vero a cui mi riferisco è del secondo tipo ed include la violenza sessuale, i danni che provoca, i codici che definiscono reato quella violenza, e le procedure che lo provano. Judith Herman e Marinella Malacrea scrivono pagine vigorose per far sì che quel vero - il delitto che è accaduto, che ha fatto male, e che magari viene negato per tutelare gli interessi o i pregiudizi di altri - sia riconosciuto: ${ }^{114}$ 'remembering and telling the truth 
about terrible events', dice la prima, 'are prerequisites both for the restoration of the social order and for the healing of individual victims'. ${ }^{115}$ Pochi, credo, vorranno opporsi a quest'esigenza.

È allora un errore rifarsi al vero, o il superamento di un pregiudizio, o non è piuttosto una necessità dovuta all'importanza che le riflessioni terapeutiche, legali e morali assumono nella letteratura contemporanea sull'abuso sessuale dei minori? Propenderei per l'ultima ipotesi: ogni testo è letto infatti in maniera diversa a seconda della rete di significati in cui lo inseriscono $\mathrm{i}$ lettori che di volta in volta ha, ${ }^{116}$ e noi, in genere, ci raccontiamo storie di violenza sessuale perché vogliamo capire che cosa provoca quegli episodi, e magari ridurne il numero; sapere quali sono i danni, e magari trovare dei modi per alleviarli; scoprire quali sono i comportamenti di una comunità responsabile, e magari adottarne alcuni o perfezionarli. La vita attorno a noi è alla base del nostro interesse e quell'interesse s'intreccia al significato che attribuiamo alla storia e alla maniera in cui la valutiamo. È inevitabile che la verosimiglianza abbia un'importanza considerevole in questo filone letterario.

È molto bene, infine, che le storie di cui ci occupiamo siano raccontate anche perché le vicende di abuso sessuale diventano grazie a loro visibili, parte integrante di una 'socially validated reality', ${ }^{117}$ e ragazzi e ragazze come quelle di cui parla la Kitzinger nell'epigrafe si ritrovano in una realtà che è in qualche misura condivisa; vi si capiscono e sono capiti. È necessario però che tali storie introducano a quella realtà per preparare ad essa, e non ne provochino una comprensione illusoria. Facendo ora l'una ora l'altra cosa i testi di Venturi e Ghinelli ribadiscono il bisogno di non adagiarsi su nessun presupposto quando affrontiamo argomenti così impegnativi.

1 Jenny Kitzinger, Framing Abuse: Media Influence and Public Understanding of Sexual Violence against Children (Ann Arbor: Pluto, 2004), p. 2. La versione originale di questo saggio usava in alcuni casi l'articolo 'la' per riferirsi a Maria Venturi e Lorenza Ghinelli (la Venturi, la Ghinelli). Le editor dell'Italianist mi hanno chiesto di evitarlo e ho accettato la loro proposta con un po' di disagio. Ho accettato perché condivido in pieno il bisogno di avversare ogni presupposto culturale che promuove forme di esclusione o discriminazione. Con un po' di disagio perché i sottintesi del linguaggio sono molti e s'intrecciano in modo complicato e persino contraddittorio. Nel variopinto gruppo di italiani che frequento la rimozione di quell'articolo ha una connotazione elitaria che a volte è solo presunta ma che altre volte è innegabile. Visto che questo saggio auspica un dialogo paritario fra critici professionistici e lettori di libri di consumo, avrei preferito pubblicarlo usando un linguaggio intermedio anche in questo senso. 
${ }^{2}$ Francesco Puccinotti, Lezioni di medicina legale, edizione seconda, 2 voll. (Macerata: ManciniCortesi, 1835), II, p. 240.

${ }^{3}$ Si veda I reati sessuali, i reati di sfruttamento dei minori e di riduzione in schiavitù per fini sessuali, a cura di Franco Coppi (Torino: Giappichelli, 2007), in particolare le pp. 1-31.

${ }^{4}$ Annarita Buttafuoco, Le mariuccine (Milano: Angeli, 1988), p. 114.

${ }^{5}$ Brian Corby et al., Child Abuse (Maidenhead: Open University Press, 2012), p. 203.

${ }^{6}$ Katherine D. Watson, Forensic Medicine in Western Society (Londra: Routledge, 2011), pp. 126-27.

7 Judith Lewis Herman, Trauma and Recovery (Londra: Pandora, 1994), pp. 28-32.

8 Philip Jenkins, Moral Panic: Changing Concepts of the Child Molester in Modern America (New Haven: Yale University Press, 1998), pp. 121-25.

9 Jenny Kitzinger, Framing Abuse: Media Influence and Public Understanding of Sexual Violence against Children (Londra: Pluto Press, 2004). Sulla situazione in Italia si vedano Marco Catino, 'Il circo mediatico-giudiziario', Problemi dell'informazione, 27.4 (2003), 515-30, e Luigi Ferrarella, 'Proposta minoritaria di ecologia giornalistica', Problemi dell'informazione, 31.4 (2006), 439-50.

10 Renato Fucini, Le veglie di Neri (Firenze: Barbera, 1882), ora disponibile nel sito https://www.liberliber.it/online/autori/autori-f/renato-fucini-alias-neri-tanfucio/le-veglie-dineri/, pp. 27-30; Giovanni Verga, Novelle rusticane [Torino: Chiantore, 1883], in Tutte le novelle, 2 voll. (Milano: Mondadori, 1983), I, 319-25. Tutte e due le novelle risalgono al 1882, ma le Novelle rusticane di Verga (che contengono 'Libertà') uscirono nell'anno successivo.

${ }^{11}$ Lorenzo Greco, Dubbiosi disiri: Famiglia e amori proibiti nella narrativa italiana fra '800 e'900 (Pisa: Giardini, 1984) è utile in particolare per Deledda e Moravia. Non mi pare che questo filone della letteratura italiana sia stato studiato in quanto tale da allora. Sono però interessanti le recensioni e gli studi su singoli testi letterari dedicati all'abuso sessuale dei minori: penso ad esempio a Christina Siggers Manson, 'In Love with Cecchino. Opening the Door to Violence in Dacia Maraini's Colomba and Voci', Journal of Romance Studies, vol. 5 (2005), n. 2, pp. 91-102; Paola Azzolini, 'll silenzio del bosco tagliato: lettura di Maria Zef', in Paola Drigo settant'anni dopo a cura di Beatrice Bartolomeo e Patrizia Zambon, Pisa, Serra, 2009, pp. 237-250; e Paola Benchi, Conversations with the Maternal: A Study of Feminist Theories and Contemporary Irish and Italian Women's Narrative, tesi di PhD, Dublino, University College Dublin, 2014.

${ }^{12}$ Si vedano Luciano Parisi: 'Narratives of child sexual abuse in Cristina Comencini's novel La bestia nel cuore', The Modern Language Review, 109.3 (2014), 676-90, e 'Proibizione e pericolo in Terza persona singolare', Modern Language Notes, 131 (2016), 235-50.

${ }_{13}$ Unfitting Stories, a cura di V. Raoul et al. (Waterloo: Wilfrid Laurier University Press, 2007).

${ }^{14}$ Arthur W. Frank, The Wounded Storyteller (Chicago: The University of Chicago Press, 1995).

${ }^{15}$ Nancy Mairs, 'Foreword', in G. Thomas Couser, Recovering Bodies: Illness, Disability and Life Writing (Madison: The University of Wisconsin Press, 1997), pp. ix-xiii (p. x). Senza nominare Mairs, Hannah Thompson critica definizioni come la sua per la connotazione immediatamente negativa che danno della condizione di disabile o di malato, in Reviewing Blindness in French Fiction 1789-2013 (Londra: Palgrave Macmillan, 2017), in particolare p. 47.

${ }^{16}$ Anne Hunsacker Hawkins, Reconstructing Illness: Studies in Pathography (West Lafayette: Purdue University Press, 1993); Peter D. Kramer, 'The Anatomy of Melancholy', New York Times Book Review, 8 aprile 1996, p. 27. 
${ }^{17}$ I testi più significativi di Venturi- sono stati scritti tutti nel o dopo il 2000: Incantesimo (Milano: RCS, 2000), Butta la luna (Milano: RCS, 2004) e La bambina perduta (Milano: RCS, 2005).

18 ; Lorenza Ghinelli, La colpa (Roma: Newton Compton, 2012). Ho citato i dati Butta la luna nella nota che precede.

19 http://opac.sbn.it/opacsbn, ora https://opac.sbn.it/opacsbn/opac/iccu/free.jsp (consultati il 24 giugno 2016 e il 26 luglio 2019). I siti delle due autrici, citati in seguito, documentano il loro successo. L'ufficio stampa della Rizzoli riferisce che Venturi ha venduto circa due milioni di copie: ' 1 milione di copie in Rizzoli e 1 milione di copie in BUR' (email personale del 13 luglio 2018). La Newton Compton non fornisce dati sulle vendite ma ha confermato che 'La colpa ha avuto grande successo, è stato per settimane nella classifica dei libri più venduti ed è entrato nella cinquina del Premio Strega' (email personale del 25 febbraio 2019).

20 Wulf Kansteiner e Todd Presner, 'Introduction' to Probing the Ethics of Holocaust Culture (Cambridge: Harvard University Press, 2016), pp. 1-42 (p. 25).

${ }^{21}$ L'espressione è di Suzanne Keen, Empathy and the Novel (Oxford: Oxford University Press, 2007), pp. 38-39. La ghettizzazione è testimoniata invece dalla quasi totale assenza di studi critici sui testi di Venturi e Ghinelli. Alla seconda sono dedicati alcuni paragrafi nell'introduzione di Caterina Mongiat Farina, "La creatura più alta del regno vivente": storie di formazione e disumanità contemporanea in "Acciaio" di Silvia Avallone', Forum Italicum, 48.1 (2014), 67-82.

22 Si vedano in particolare Roger C. Schank, Tell Me a Story (New York: Scribner's Sons, 1990), p. 10, e Jerome Bruner, Acts of Meaning (Cambridge: Harvard University Press, 1990), pp. 39-52, 67-97, 137-38.

${ }^{23}$ Marie Keenan, Child Sexual Abuse and the Catholic Church: Gender, Power and Organizational Culture (Oxford: Oxford University Press, 2011), pp. xx-xxii (p. xxii).

${ }^{24}$ Arthur W. Frank, At the Will of the Body (Boston-New York: Houghton Mifflin, 2002), p. 81.

${ }^{25} \mathrm{Si}$ vedano in particolare Alberto Moravia, Le ambizioni sbagliate, in Opere, vol. 1, Romanzi e racconti 1927-1940, a cura di Francesca Serra (Milano: Bompiani, 2000), pp. 473-1009, e Giacoma Limentai, Trilogia. (Roma: lacobelli, 2013).

26 The Wounded Storyteller, pp. 115-36. Anche lo schema di Frank è tripartito: a volerlo seguire, le storie di Venturi e Ghinelli apparterrebbero per lo più alla restitution narrative (pp. 75-96) e quelle di Moravia alla chaos narrative (pp. 97-114).

27 Umberto Eco, Il superuomo di massa: Retorica e ideologia nel romanzo popolare (Milano: Bompiani, 1978), pp. 7-18. Diana Holmes, 'The mimetic prejudice: the popular novel in France', in Imagining the Popular in Contemporary Popular Culture, a cura di D. Holmes e David Looseley (Manchester: Manchester University Press, 2013), pp. 85-122, critica una simile contrapposizione 'between an inferior literature read by the many, and an enriching, progressive literature read by the few' (p. 116) da lei individuata nella cultura francese contemporanea.

${ }^{28}$ La rappresentazione dei rivoluzionari nei Demoni (Torino: Einaudi, 1942) [1872] di Dostoevskij è degna anche per questo di seria considerazione.

${ }^{29}$ Nina Schmidt, The Wounded Self: Writing Illness in Twenty-First-Century German Literature (Rochester: Camden House, 2018), pp. 16-21, è eloquente su questo punto

30 'I modelli astratti [...] del romanzo problematico e del romanzo consolatorio sono appunto dei modelli, e le singole opere appaiono poi effetto di numerose contaminazioni e ciascuna risolve i problemi a modo proprio' (Eco, p. 18). 
31 Tzvetan Todorov osserva che 'le lecteur ordinaire, qui continue de chercher dans les œuvres qu'il lit de quoi donner sense à sa vie, a raison contre les professeurs, critiques et écrivains qui lui disent que la littérature ne parle que d'elle même, ou qu'elle n'enseigne que le désespoir', $L a$ littérature en peril (Parigi: Flammarion, 2007), p. 72.

32 Laura Finley, Domestic Abuse and Sexual Assault in Popular Culture (Santa Barbara: Praeger, 2016). L'espressione 'popular culture' nel libro è usata in modo vago: quella cultura include infatti opere di Pedro Almodovar, Vladimir Nabokov, Joyce Carol Oates, Wim Wenders e Tennessee Williams che molti non considererebbero 'popolari'.

33 Maria Venturi, Caro amore (Milano: Rizzoli, 1988); M. Venturi, L'amore s'impara (Milano: Rizzoli, 2011).

${ }^{34}$ Una di quelle serie, Incantesimo (In), è andata in onda tra il 1998 e il 2008 in dieci stagioni su Rai 1 e Rai 2. Utili informazioni sulla scrittrice apparivano nel sito dedicato a Come salvare un amore, http://www.comesalvareun amore.it/biografia.shtml (consultato il 6 marzo 2015 e ora dismesso). Una picola parte è ancora disponibile in https://www.rizzolilibri.it/autori/mariaventuri/ (consultato il 26 luglio 2019)

${ }^{35}$ In, p. 5

36 Nessun altro narratore contemporaneo che conosco, per esempio, ha trattato con pari attenzione del lavoro degli assistenti sociali in Italia.

37 Mc, p. 202.

38 Annamaria Trevale, 'Niente è per caso: incontro non convenzionale con Maria Venturi', Sul romanzo, http://www.sulromanzo.it/blog/niente-e-per-caso-incontro-non-convenzionale-conmaria-venturi (consultato il 26 luglio 2019). L'articolo di Eletta Flocchini, 'Maria Venturi scrittrice con il cuore', Corriere della sera, 14 febbraio 2015, p. 13, contiene una serie di interessanti dichiarazioni della scrittrice sulla propria poetica.

${ }^{39} \mathrm{Mc}$, p. 62.

${ }^{40}$ Maria Venturi, La moglie nella cornice (Milano: RCS, 1987).

${ }^{41}$ Bl, p. 58.

42 Mc, p. 85.

${ }^{43}$ Mc, p. 21.

${ }^{44}$ Mc, p. 17.

${ }^{45} \mathrm{Mc}, \mathrm{p} .182$.

${ }^{46}$ Marinella Malacrea ricorda che metà delle tossicodipendenti italiane a condotta deviante sono state vittime di abuso sessuale, spesso prima dei tredici anni; 'Caratteristiche, dinamiche ed effetti della violenza su bambini e bambine', in Vite in bilico, a cura di Donata Bianchi e Ermenegildo Ciccotti (Firenze: Istituto degli innocenti, 2006), pp. 3-72 (pp. 7 e 30).

47 Valeria Giamundo e Valentina Capobianco, 'L'abuso sessuale', in Abuso e maltrattamento all'infanzia, a cura di V. Giamundo (Milano: Angeli, 2013), pp. 330-99 (p. 357).

48 Maura Anfossi, 'Riflessioni su esperienze di sostegno a vittime di abuso', La famiglia, 231 (1995), 29-44.

49 Marinella Malacrea, Trauma e riparazione (Milano: Cortina, 1998), pp. 34-35.

50 Maria Venturi, La storia spezzata (Milano: RCS, 1989), p. 95.

51 Trauma, p. 15. L'immagine della bomba a orologeria pronta a dilaniare ricorre con frequenza negli scritti sui bambini maltrattati o terrorizzati: la si trova anche in Bruno Bettelheim, The Uses 
of Enchantment (Harmondsworth: Penguin, 1978), p. 31, e in Louise Armstrong, Kiss Daddy Goodnight: A Speak-Out on Incest (New York: Hawthorn, 1978), p. 27.

52 'Narratives of illness are often somber, but they need not be depressing. Autobiographical narratives, almost by definition, involve comic plots; that is, the protagonist is better off at the end than at the beginning. Memoirs of others' illnesses tend to find some redeeming significance even in terminal illness', G. Thomas Couser, p. 16.

53 Paola Drigo, Maria Zef (Milano: Treves, 1936). Il testo è disponibile nel sito https://www.liberliber.it/mediateca/libri/d/drigo/maria_zef/pdf/drigo_maria_zef.pdf, p. 220 (consultato il 26 luglio 2019). Giacoma Limentani, Trilogia (Roma: lacobelli, 2013), p. 261. La prima parte di questo libro, In contumacia, è stata pubblicata come opera a se stante nel 1967 dall'editore Adelphi di Milano; la seconda parte, Dentro la D, nel 1992 dall'editore Marietti di Casale Monferrato; e la terza (da cui proviene la citazione), La spirale della tigre, nel 2003 dall'editore Giano di Varese.

54 Per un'analisi dettagliata si veda Luciano Parisi, 'Il silenzio di Paola Drigo', Rivista di letteratura italiana, 34.1 (2016), 77-91.

55 Valerie Raoul et al. 'Introduction: Aesthetics, Authenticity, and Audience', in Unfitting Stories, pp. 25-31 (p. 26).

56 Platone, La repubblica, X, 604-605; Jean-Marie Schaffer, Pourquoi la fiction? (Parigi: Seuil, 1999), pp. 34-60.

57 Jerome Bruner, Actual Minds, Possible Worlds (Cambridge: Harvard University Press, 1986), pp. 11-12 (p. 12).

58 Si vedano Frank Kermode, The Genesis of Secrecy: On the Interpretation of Narrative (Cambridge: Harvard University Press, 1979), pp. 72 e 84; Martin Jay, 'Of Plots, Witnesses and Judgments', in Probing the Limits of Representation a cura di Saul Friedlander (Cambridge: Harvard University Press, 1992), pp. 97-107, e Dominick LaCapra, 'Representing the Holocaust', ivi, pp. 108-27; e Theodor Adorno, 'Impegno', in Note per la letteratura (Torino: Einaudi, 2012), pp. 141-60.

59 'We don't blame [art] for the uses to which it is put. We don't expect it to intervene, to determine, to make peace or war, to ebb poverty, or greed, to abate suffering. We ask only that it be beautiful and true. We turn to it to dignify our experience of the world', Rachel Cusk, The Last Supper (Londra: Faber \& Faber, 2010), p. 177.

${ }^{60}$ Couser, p. 41.

${ }^{61}$ Alberto Moravia, Le ambizioni sbagliate (Milano: Mondadori, 1935).

62 Ian McEwan, Atonement (New York: Anchor Books, 2001), p. 350.

63 'Fictions do not work if they fail to confront danger, horror [and] loss' (Holmes, p. 95).

${ }^{64}$ Adorno, p. 153.

${ }^{65}$ Frank, At the Will, pp. 136-38.

${ }^{66} \mathrm{Bp}$, pp. 14-15.

67 Bp, p. 57.

68 Bp, p. 11.

${ }^{69} \mathrm{Bp}$, pp. 26 e 44.

70 Bp, p. 48. 
71 II filone di cui ci occupiamo smentisce ripetutamente il preconcetto che i lettori italiani di romanzi di successo siano svagati. Esistono testi sull'abuso sessuale dei minori che insistono su dettagli scabrosi in vista di un sentimentalismo patetico o di un voyeurismo contrabbandato ma, grazie alla responsabilità della maggior parte dei lettori, il loro successo commerciale è stato finora minimo come quello critico.

72 In, pp. 177 e 182.

73 In, p. 182.

${ }^{74}$ Bl, p. 174.

${ }^{75}$ Alberto Moravia, L'attenzione (Milano: Bompiani, 1965) e A. Moravia, La vita interiore (Milano: Bompiani, 1978).

${ }^{76}$ Bl, pp. $175-76$.

77 Bl, p. 178.

78 La mente del ragazzo 'era stata ottenebrata da un raptus di follia oppure la sua mano aveva colpito e colpito e colpito spinta dall'odio per una piccola vita felice e innocente?'. Che cosa lo aveva poi 'spinto a uccidere se stesso? Un barlume di luce, un soprassalto di pentimento, la rabbiosa volontà di ferire a morte gli Alfonsi, una sfida estrema per richiamare a sé l'attenzione?' (Bl, pp. 175-76).

${ }^{79}$ Maria Venturi, In punta di cuore (Milano: RCS, 1995).

${ }^{80}$ Maria Venturi, L'amore stretto (Milano: RCS, 1998).

${ }^{81}$ Caro amore, p. 49.

${ }^{82}$ Alcuni antropologi e psicologi parlano di 'illusioni positive'. Si vedano Lionel Tiger, Optimism: the Biology of Hope (Londra: Secker \& Warburg, 1979), e Shelley Taylor, Positive Illusions: Creative Self-Deception and the Healthy Mind (New York: Basic Books, 1989).

${ }^{83}$ Per illustrare questo punto Laura Finley rimanda a una canzone di Bessie Smith, Outside of That (Columbia, 1923): 'I've got the meanest man in the land / But his love is best, thick and grand / [...] People say I'm a fool / He's heartless and also cruel. / But outside of that, he's all right with me. / Outside of that, he's sweet as he can be' (Finley, Domestic Abuse and Sexual Assault In Popular Culture, p. 5). Si veda anche C. Randall Colvin e Jack Block, 'Do Positive Illusions Foster Mental Health?', Psychological Bulletin, 116.1 (1994), 3-20.

84 Queste e molte altre informazioni sono disponibili in https://www.newtoncompton.com/autore/lorenza-ghinelli https://www.rizzolilibri.it/autori/lorenza-ghinelli/ (consultati il 26 luglio 2019).

${ }^{85} \mathrm{Si}$ veda la recensione anonima, 'Un incubo molto intrigante', apparsa sul Riformista del 5 febbraio 2001, p. 13.

${ }^{86}$ Le citazioni provengono, in ordine, da La colpa, pp. 92, 136, 343, 47, 191, 12, 36, 43, 18 e 130. Ranieri Polese, 'Ritratto al nero di tre adolescenti', Corriere della sera, 14 febbraio 2012, p. 36, sostiene che le parti migliori de La colpa 'restano quelle delle allucinazioni scandite su parole e musica dell'ultimo rock'.

87 Lorenza Ghinelli, Daniele Rudoni e Simone Sarasso, J.A.S.T.: Just Another Spy Story, 3 voll. (Venezia: Marsilio, 2010), p. 8.

88 J.A.S.T, III, pp. 60-64 e 66-69.

89 J.A.S.T, III, p. 75.

${ }^{90} \mathrm{Cp}$, p. 44. Sergio Pent, 'Un corvaccio nero vola sui ragazzi di Rimini', Tuttolibri, 4 febbraio 2012, p. 7, parla di un 'linguaggio aspro e telematico, frenetico come un SMS'. 
${ }^{91} \mathrm{Cp}$, p. 64.

${ }^{92}$ Cp, pp. 10-11. Micol Argento, 'Il dolore dell'infanzia', La repubblica, 3 aprile 2012, p. 12, osserva che 'le atmosfere sono solo abbozzate, ma la lingua le rende taglienti'.

93 Lorenza Ghinelli, Con i tuoi occhi (Roma: Newton Compton, 2013).

${ }^{94}$ Franco Pezzini, 'La colpa', L'indice dei libri del mese, maggio 2012, p. 44, insiste a ragione su 'la sincerità partecipe' con cui la scrittrice descrive le incertezze di questi giovani.

95 La scrittrice, per esempio, ricorre sempre alla stessa situazione per segnalare la rabbia irrisolta del ragazzo abusato: lo zio stupra Martino mentre il registratore suona una canzone dei Clash $(C p$, pp. 42-44 e 161); da allora Martino è violento ogni volta che qualcuno suona un motivo di quel gruppo - quando un amico arriva con un'auto nuova, che Martino prende a pugni ( $C p, p$. 12); quando Martino spacca il setto nasale a chi seleziona 'Guns on the Roof' in un bar ( $C p, p .90)$; e poi durante una gita ( $C p$, p. 91); e in discoteca ( $C p$, p. 142).

${ }^{96}$ Ernesto Caffo, 'Prefazione alla seconda edizione', in Criteri di valutazione nell'abuso all'infanzia a cura di E. Caffo et al. (Milano: McGraw-Hill, 2004), pp. xv-xvii.

97 Ct, p. 27.

${ }^{98} \mathrm{Ct}$, p. 109.

${ }^{99} \mathrm{Ct}, \mathrm{p} .107$.

100 Ct, p. 107.

101 Ct, p. 264.

102 Ct, p. 290.

${ }^{103} \mathrm{Ct}$, p. 353.

104 Ct, p. 353.

${ }^{105} \mathrm{Ct}$, pp. 368-69.

106 Lev Tòlstoj, Tutti i racconti, 2 voll. (Milano: Mondadori, 1991), I, p. 8.

107 Si vedano anche le riflessioni di Peter Stamm, in Deborah Treisman, 'This Week in Fiction: Peter Stamm', The New Yorker, 3 maggio 2012, disponbile in https://www.newyorker.com/books/page-turner/this-week-in-fiction-peter-stamm (consultato il 26 luglio 2019).

108 Bruno Bettelheim, The Uses of Enchantment: The Meaning and Importance of Fairy Tales (Harmondsworth: Penguin, 1978), p. 133.

109 La distinzione tra ermeneutica del sospetto ed ermeneutica della cooperazione, proposta inizialmente da Paul Ricoeur, è stata ripresa da Rita Felski, The Limits of Critique (Chicago: University of Chicago Press, 2015), pp. 1-39. Ne propongo una versione adatta al materiale di cui tratto.

110 Paul Ricoeur, Time and Narrative (Chicago: University of Chicago Press, 1984), p. 47; Jerome Bruner, Making Stories (Cambridge: Harvard University Press), pp. 63-87.

111 Tzvetan Todorov, La poétique de la prose (Parigi: Seuil, 1971), pp. 92-99 (p. 92).

112 Denis Pernot, 'Vraisemblance', in Le dictionnaire du littéraire, a cura di Paul Aron et al. (Parigi: Presses Universitaires de France, 2008), pp. 626-27 (p. 627).

${ }^{113}$ Si veda l'introduzione senza titolo di Yves Le Bozec al numero speciale, intitolato Le Vrai et le Vraisemblable e a cura dello stesso Le Bozec, della Revue des sciences humaines, 4 (2005), 7-8. 114 Herman, Trauma and Recovery, pp. 7-32; Malacrea, Trauma e riparzione, pp. 85-127. [I am not sure I understood your indication here and suddenly I cannot add comments]

115 Herman, p. 1. 
${ }^{116}$ Si vedano in proposito Hans-Georg Gadamer, Wahrheit und Methode (Tübingen: Mohr, 1960), e Jens Zimmermann, Hermeneutics (Oxford: Oxford University Press, 2015).

117 Herman p. 8. 